\title{
$\pi$-Stacked polyphenolic dimers: A case study using dispersion-corrected methods
}

\author{
I. Bayach ${ }^{1}$, J. C. Sancho-García ${ }^{2,3 *}$ F. Di Meo ${ }^{2}$, \\ J.-F. F. Weber ${ }^{4}$, and P. Trouillas ${ }^{1,3,5 \dagger}$ \\ ${ }^{1}$ Institut National de la Santé Et de la Recherche Médicale, UMR-S850, \\ Faculté de Pharmacie, \\ Université de Limoges, 87025 Limoges, France \\ 2 Departamento de Química Física, \\ Facultad de Ciencias, \\ Universidad de Alicante, 03080 Alicante, Spain \\ ${ }^{3}$ Laboratory for Chemistry of Novel Materials, \\ University of Mons, 7000 Mons, Belgium \\ ${ }^{4}$ Atta-ur-Rahman Research Institute for Natural Product Discovery, \\ Faculty of Pharmacy, \\ Universiti Teknologi MARA, 42300 Puncak Alam, Malaysia \\ ${ }^{5}$ Department of Physical Chemistry, \\ Faculty of Science, \\ Palacky University, 77146 Olomouc, Czech Republic
}

May 27, 2013 
${ }^{*}$ E-mail: jc.sancho@ua.es
${ }^{\dagger}$ E-mail: patrick.trouillas@unilim.fr 


\begin{abstract}
The accuracy of dispersion-corrected calculations (DFT-D2, DFTD3 and DFT-NL) is assessed here, with large basis sets (def2-QZVP) to avoid incompleteness effects, for the most stable structure of a realworld polyphenol dimer chosen as an appropriate model. Natural polyphenols form such complexes with $\pi$-stacking playing a key stabilizing role. Our benchmark calculations predict its existence favoured by $22-24 \mathrm{kcal} / \mathrm{mol}$ with respect to the isolated monomers, mainly driven by both $\pi-\pi$ and H-bonding interactions. The adequate comparison of lower-cost DFT-based methods allowed bracketing their expected accuracy. These results thus pave the way towards reliable studies of challenging aggregation processes of natural products.
\end{abstract}




\section{Introduction}

Polyphenols sensu lato [1] (e.g., lignans and lignins, chalconoids and flavonoids, condensed and hydrolysable tannins, phlorotannins, depsides, stilbenoids, curcuminoids, anthraquinoids, etc.) constitute one of the most important groups of natural products, with some $10^{5}$ defined structures [2]. They have been isolated from all plant organs (e.g., bark, wood, roots, leaves, flowers, fruit, and seeds) in which they may accumulate in substantial amounts. Therefore, they are quite abundant in human diets (e.g., fruit, vegetables, spices and beverages) exhibiting various potential health benefits (see for example [3] and [4]). In order to fully rationalize and increase these beneficial effects, particular attention is paid to the chemical properties involved in e.g., (i) their biological properties and (ii) their biomimetic syntheses. Non-covalent complexes have been suggested in last decades to play an important role in these chemical properties. The high $\pi$-delocalization observed in the polyphenol backbone would allow monomer self-association, while the presence of $\mathrm{OH}$ substituents allows additional formation of strong intermolecular H-bonds. For example, non-covalent interactions in polyphenols lead to complexes involved in many key natural processes including: (i)

plant color persistence $[5,6]$; and (ii) regio- and stereoselective biogenetic reactions (see for example [7]). However, the definite role of these weak interactions in polyphenol compounds is still under scrutiny; theoretical predictions are thus of (expectedly) high value for the rationalization of these processes and they appear as the only current way to provide a direct molecular visualization of these non-covalent dimers in solution. The theoretical results thus appear issued from a "computational microscope" supporting the indirect experimental visualization (e.g., bathochromic shift observed in $\mathrm{UV} / \mathrm{Vis}$ absorption measurements). 
A small yet emblematic group of polyphenols is that of oligostilbenoids, biogenetically deriving from the oligomerisation of polyhydroxylated stilbene precursors. The large variety of their chemical structures allows for a broad range of biological activities including antibacterial, antioxidant, anti-fungal and anti-inflammatory properties [8]. Oligostilbenoid polymerization is a typical case where the importance of non-covalent interactions has been recently highlighted: regio- and stereoselective synthesis is largely driven by the ability of these compounds to self-assembly in solution prior to the oxidative initiation stage $[9,10]$. The extended $\pi$-delocalization of $\epsilon$-viniferin derivative (Figure 1) is known to allow long-range interactions in solution $[9,11]$, as it is indeed confirmed by NMR-based dynamical and structural studies [12]. Therefore, if theory aims at describing a complete picture of these interactions and corresponding supramolecular association at the molecular scale, the treatment of weak interactions is mandatory. Note also that regarding the large size of the (if any) dimer involved precludes the use of methods that unfavourably scale with system size. Thus, Density Functional Theory (DFT) becomes the favoured and more judicious choice here, also keeping in mind that such methodology should be used for large series of compounds similar in size, as a predictive tool in the near future. Taken into account these issues, the present study deals with structure and energetics of the non-covalent dimer of $\epsilon$-viniferin, which is used as a prototype to tackle as accurately as possible these effects leading to dimerization. Various DFT functionals including dispersive effects are used and the associated association energies are compared to reference resutls. To present the achievements towards the above goals, the manuscript is structured as follows: Section 2 presents the different DFT-based theoretical methods able to largely deal 
with non-covalent interactions at both intra- and intermolecular levels. Section 3 reports the careful application of these methods concomitantly with their benchmarking. This would then allow reaching predictive yet robust conclusions about the stability of this kind of complexes, and complementarily shedding light about the possible routes followed in their reactivity.

\section{Theoretical methods}

\subsection{Modelling dispersion effects}

Dispersion physics arises from locally induced interactions, be them intraor intermolecular, after the response of the electronic cloud in one region to the presence of instantaneous and fluctuating charge densities in another [13]. In other words, whenever polarizable electronic clouds are present in two spatially separated but interacting fragments or subsystems, even if weakly overlapping, these correlated dipole-dipole interactions might clearly drive self-assembly or supramolecular organization. To account for these interactions is a real challenge for any theoretical method currently in use. A purely $a b$ initio treatment would therefore imply the use of energy magnitudes depending simultaneously of properties at two separate points in space $\mathbf{r}$ and $\mathbf{r}^{\prime}$. This is one of the reasons why classical Møller-Plesset perturbation theory truncated at second order (MP2) is able to partly capture the physics behind these interactions. It has been considered as the pioneering yet simplest theoretical method to be applied within this context. Contrarily to this, unmodified or poorly fitted DFT-based functionals completely fails due to the short-sight treatment of matter imposed by the dependence of common (semi-local) functionals on the density $(\rho)$ and its gradient $(\nabla \rho)$ on $\mathbf{r}$ exclusively. Here we briefly review the most common DFT-based dispersion methods currently applied [14] to overcome this undesired but generalized 
drawback.

Due to the difficulty to self-consistently introduce the dispersion energy $\left(E_{\mathrm{D}}\right)$ into the computational treatment, one normally adds this contribution to the electronic energy in a post self-consistent way: $E_{\mathrm{DFT}+\mathrm{D}}=E_{\mathrm{DFT}}+E_{\mathrm{D}}$, the density thus remains unaffected upon the dispersion treatment. The modelling of $E_{\mathrm{D}}$ term is based on the well-known pairwise additivity of effects between atoms $A$ and $B$ belonging to weakly overlapping fragments:

$$
E_{\mathrm{D}}=\sum_{B>A}^{N}\left[-\frac{C_{6}^{A B}}{R_{A B}^{6}}-\frac{C_{8}^{A B}}{R_{A B}^{8}}-\frac{C_{10}^{A B}}{R_{A B}^{10}} \ldots\right]
$$

where $C_{A B}^{j}$ are interatomic dispersion coefficients and $R_{A B}$ is the distance between the two atoms involved. The simplest approach, coined as D2 [15] truncates the expansion at first order providing the $1 / R^{6}$ attractive term as found in the classical Lennard-Johnes potential. This term is however weighted introducing a functional-dependent parameter $\left(s_{6}\right)$ to efficiently couple both terms, $E_{\mathrm{DFT}}$ and $E_{\mathrm{D}}$ :

$$
E_{\mathrm{D} 2}=-s_{6} \sum_{B>A}^{N} \frac{C_{6}^{A B}}{R_{A B}^{6}} f\left(R_{A B}\right),
$$

also relying on a damping function, $f\left(R_{A B}\right)$, to efficiently and more physically switch from the infinite separate limit to distances belonging to the binding region [16]. This correction has been successfully applied for complexes of the most interest [17-19], although a more sophisticated correction (D3) has been recently introduced to overcome some known limitations of the latter [20]. In this case the correcting term is given by:

$$
E_{\mathrm{D} 3}=-\sum_{B>A}^{N} \sum_{n=6,8} s_{n} \frac{C_{n}^{A B}}{R_{A B}^{n}} f_{n}\left(R_{A B}\right),
$$

expanding the former series and introducing now $n$ th-order dispersion coefficients allowing a better respond to changes in chemical environment. The 
mathematical form imposed to the damping function introduces two new parameters, $s_{r, n}$, to be defined for each value of $n$,

$$
f_{n}\left(R_{A B}\right)=\frac{1}{1+6\left(\frac{R_{A B}}{s_{r, n} R_{0}^{A B}}\right)^{-\alpha}},
$$

with the ratio $R_{0}^{A B}=\sqrt{\frac{C_{8}^{A B}}{C_{6}^{A B}}}$. Note that the damping function reduces to a simpler form for DFT-D2 and that more details about the form and (expectedly) negligible influence of other devised damping functions can be found in Ref. [21].

Interestingly, there is a recent renewed interest [22-24] to obtain the dispersion energy directly from the electron density through a non-local (NL) correlation functional which inherently account for this contribution. The total energy is now $E_{\mathrm{DFT}+\mathrm{D}}=E_{\mathrm{DFT}}+E_{\mathrm{NL}}$, with $E_{\mathrm{NL}}$ being a correction covering mostly long-ranged interactions between these instantaneous and fluctuating induced local dipoles:

$$
E_{\mathrm{NL}}=\int d \mathbf{r} \rho(\mathbf{r})\left[\beta+\frac{1}{2} \int d \mathbf{r}^{\prime} \rho\left(\mathbf{r}^{\prime}\right) \Phi\left(\mathbf{r}, \mathbf{r}^{\prime}\right)\right]
$$

using the specific construction called VV10 [25] for the $\Phi\left(\mathbf{r}, \mathbf{r}^{\prime}\right)$ kernel. Note that in the NL-approach a short-range attenuation functional-dependent parameter dubbed $b, \beta=\beta(b)$, is required to efficiently couple the total correlation energy to any particular exchange form used. Note also that a (more costly) double integration is required for $E_{\mathrm{NL}}$, which implies the use of an additional numerical grid on top of the grid used for the local exchangecorrelation functional; however, thanks to recent techniques [26] this step is not a bottleneck for real calculations. 


\subsection{Technical details}

The choice of the exchange-correlation functionals BP86 and B3P86 is motivated by two features: (i) the excellent performance shown by the latter model for bond dissociation energies [27-29] and optoelectronic properties [30] of many different polyphenols; and (ii) the lower computational cost of the parent non-hybrid model (BP86), which would allow applications to larger real-world systems and large series, due to the pervasive trade-off between accuracy and computational resources. The related parameters of eqs. (2)-(4) are reported in Table 1. Whereas these are taken from Grimme's work [20] for BP86 (-D2 or -D3), we have recently extended the B3P86 model (-D2) in this way [5]. The attenuation parameter $b$ required for the use of both models together with eq. (5), BP86-NL and B3P86-NL, is also assessed here for the first time (to the best of our knowledge) in order to obtain (vide infra) its optimum value (Table 1 ).

All calculations were performed with the ORCA program [31] with the built-in def2-xVP family of basis sets, unless otherwise noticed, i.e. the hierarchy def2-SVP, def2-TZVP, and def2-QZVP. The cost of the calculations was reduced in all cases by the use of the 'resolution of the identity' (RI) [32] and/or the 'chain-of-spheres' (COSX) [33] algorithms, for Coulomb or exchange integrals, respectively; note that the largest calculations performed here involve 7500 primitive basis functions. Concerning numerical grids for integration, to be on the safer side, their size was always made larger than hardwired defaults: grid4 and grid6 for computing $E_{\mathrm{DFT}}$ and $E_{\mathrm{NL}}$, respectively. Due to the (expected) flatness of potential energy curves around the equilibrium geometry, we also imposed larger-than-default thresholds for the optimization algorithm. 
The intermolecular interaction or association energy $(\Delta E)$ was calculated as the difference between the energy of the complex $\left(E_{\mathrm{CX}}\right)$ and those of the free monomers I and II $\left(E_{\mathrm{MON}-\mathrm{I}}\right.$ and $\left.E_{\mathrm{MON}-\mathrm{II}}\right)$. A negative value for $\Delta E$ thus implies the existence of the complex with respect to the pair of isolated monomers.

The basis set superposition error (BSSE), which introduces a spurious (overstabilizing the dimer formation) energetic $\Delta E$ (BSSE) contribution to $\Delta E$ was calculated by the standard counterpoise method, giving thus rise to:

$\Delta E=\left[E_{\mathrm{CX}}^{\mathrm{CX}}(\mathrm{CX})-E_{\mathrm{MON}-\mathrm{I}}^{\mathrm{MON}-\mathrm{I}}(\mathrm{MON}-\mathrm{I})-E_{\mathrm{MON}-\mathrm{II}}^{\mathrm{MON}-\mathrm{II}}(\mathrm{MON}-\mathrm{II})\right]-\Delta E(\mathrm{BSSE})$,

in which:

$$
\begin{aligned}
\Delta E(\mathrm{BSSE}) & =\left[E_{\mathrm{MON}-\mathrm{I}}^{\mathrm{CX}}(\mathrm{CX})-E_{\mathrm{MON}-\mathrm{I}}^{\mathrm{CX}}(\mathrm{MON}-\mathrm{I})\right. \\
& \left.+E_{\mathrm{MON}-\mathrm{II}}^{\mathrm{CX}}(\mathrm{CX})-E_{\mathrm{MON}-\mathrm{II}}^{\mathrm{CX}}(\mathrm{MON}-\mathrm{II})\right]
\end{aligned}
$$

where $E_{\mathrm{P}}^{\mathrm{Q}}(\mathrm{R})$ is the energy of fragment $\mathrm{P}$ calculated at the optimized geometry of $\mathrm{Q}$ and with the basis set of $\mathrm{R}$. Note how this procedure always implies an extra significant computational effort, which must be considered as a limit for further extensions of this methodology to large series of compounds. On the other hand, the BSSE can be nearly minimized, and thus the contribution $\Delta E(\mathrm{BSSE}) \simeq 0$, by applying a very large basis set, the def2-QZVP here; we will thus compare the results of both approaches to estimate the results at the Complete Basis Set (CBS) limit. 


\section{Results and discussion}

\subsection{Optimized geometry of the complex}

The geometry of the complex has been calculated at the BP86-D2, BP86D3, and B3P86-D2 levels with the def2-SVP basis set; the geometry is not expected to significantly change upon use of larger basis sets. Figure 2 shows two views of the head-to-tail complex formed, no matter the theoretical level employed: (i) a large $\pi$-stacking of the backbone upon resulting monomer interaction; (ii) a release of steric hindrance caused by the phenolic moieties acting as substituents after adopting (almost) perpendicular positions with respect to the central backbone; and (iii) the strong directionality and force of some intermolecular hydrogen bonds $\mathrm{O}-\mathrm{H} \cdots \mathrm{O}$, occurring within the two heads or at the tails; to name just a few interesting facts. The dimer intermolecular distance, defined as the closest distance between carbon atoms of the central backbone belonging to both monomers, is around 2.99, 3.02, and $2.98 \AA$, at the BP86-D2, BP86-D3, and B3P86-D2 levels, respectively. These features clearly show how the choice of the exchange-correlation functional is of relatively little importance to describe the geometrical features, once a proper correction for dispersion is considered. Whereas the backbone of isolated monomers is almost completely planar, the strong intermolecular interactions (mainly H-bonds) slightly bent the monomers in the complex geometry. These hydrogen bonds located at the edges of the backbone reduce the intermolecular distance to values that are normally repulsive (the sum of $\mathrm{C}-\mathrm{C}$ van der Waals radii is $3.5 \AA$ ); this effect has also recently been detected with halogenated polycyclic aromatic complexes [34]. 


\subsection{Reference data}

The size $(\mathrm{N})$ of the system tackled here precludes the use of highly sophisticated yet very costly $a b$ initio methods like $\operatorname{CCSD}(\mathrm{T})$ or some of its variants [35], despite recent progress [36] to reduce its formal dependence with size: $O\left(\mathrm{~N}^{7}\right)$. The so-called Spin-Component-Scaled (SCS) ${ }^{1}$ MP2 method [37] was used as the current baseline to deal with dispersion effects. Note that despite being a method scaling as $O\left(\mathrm{~N}^{5}\right)$, which dramatically alleviates the computational cost with respect to $\operatorname{CCSC}(\mathrm{T})$, this (generalpurpose) method is known to provide remarkable accuracy for a wide variety of covalent and non-covalent interacting molecular systems [38], including para-diiodobenzene [39] or anthracene [40] dimers extracted from crystalline structures and paracyclophane derivatives [41], and will be thus used as reference in the following. Note that all these single-point calculations were performed here at the BP86-D3 optimized geometry for both the complex and the isolated monomers. As expected, the calculated SCS-MP2 interaction energy decreases, upon augmenting the size of basis sets: $-21.8,-18.4$, and $-16.2 \mathrm{kcal} / \mathrm{mol}$ with the def2-SVP, def2-TZVP, and def2-QZVP basis sets, respectively. SCS-MP2 has been shown to slightly underestimate noncovalent association energies [42]; thus, within the SCS-MP2-D2 corrected method $\left(s_{6}=0.16\right.$, obtained with a large TZVPP basis set [42]), the combination is somewhat considered as an artifact dropping the values to -24.3 and $-22.1 \mathrm{kcal} / \mathrm{mol}$ with the def2-TZVP and def2-QZVP basis sets, respectively. However, the high stability of these complexes is confirmed with a new SCS-MP2 version, namely SCS-S66-MP2 [43] specifically suited for noncovalent interactions, which is additionally known to become very accurate

\footnotetext{
${ }^{1}$ This method scales differently the contribution to correlation energy arising from opposite- or same-spin contribution.
} 
in the description of strong hydrogen bonds. A $-26.5 \mathrm{kcal} / \mathrm{mol}$ energy of complexation was obtained with the def2-TZVP basis set, which serves to firmly bracket the reference value and to validate afterwards the DFT-based approximations employed.

\subsection{Assessment of DFT-based dispersion corrections}

Further reduction of computational time and associated resources will necessarily proceed through the use of DFT-based approaches scaling as much as $O\left(\mathrm{~N}^{4}\right)$, e.g. B3P86, or even as $O\left(\mathrm{~N}^{3}\right)$, e.g. BP86. The association energies obtained with BP86-D2, BP86-D3, and B3P86-D2 are compared to the reference data in Figure 3. Note first that the complex is predicted to be unbound without these -D2 or -D3 corrections, independently of the functional employed. Note also that the calculations with the large def2-TZVP and def2-QZVP basis sets were done at the def2-SVP respective optimized geometries. The association energies are always largely affected by the BSSE: the energy decreases by $7-8 \mathrm{kcal} / \mathrm{mol}$ when going from the def2-SVP to the def2-TZVP, and only by $1.5-2.0 \mathrm{kcal} / \mathrm{mol}$ upon extension to the nearly saturated def2-QZVP basis set. The use of the counterpoise correction, see eq. (7), brings the results close to those achieved by using the large def2-QZVP basis set, which can be thus considered near to the (unknown) CBS limit. As a matter of example, the counterpoise-corrected BP86-D3 association energies are $-15.8,-18.5$, and $-18.6 \mathrm{kcal} / \mathrm{mol}$, with the def2-SVP, def2-TZVP, and def2-QZVP, respectively, as compared to the values obtained without BSSE correction i.e., $-28.0,-20.9$, and $-19.1 \mathrm{kcal} / \mathrm{mol}$ for the three basis set, respectively. The underlying BSSE can be thus estimated to be -12.2 (unacceptable), -2.4 (mildly acceptable), and -0.5 (within the "chemical ac- 
curacy" range) kcal/mol, for the def2-SVP, def2-TZVP or def2-QZVP basis sets, respectively. This trend is similar to all the functionals tested here. Note also that this way to calculate the BSSE is believed to slightly overestimate its effect, and some authors even propose to scale it down by a factor between 0.5 and $1.0[44,45]$. This is the reason why in the following we will estimate (if any) the CBS limit as the average between the def2QZVP values with and without the counterpoise-correction. As a matter of example, the BP86-D3/CBS result will be thus $-18.8 \pm 0.2 \mathrm{kcal} / \mathrm{mol}$, being the error bar the difference of each method with respect to its averaged value.

The $s_{6}$ parameter entering into the B3P86-D2 form was originally assessed with the cc-pVTZ basis set for a non-covalent polyphenol dimer [5] i.e., with very similar interaction than the present dimer. The effect of using a particular family (cc-pVxZ or def2-xVP) of basis sets has been also investigated here as a by-product. The cc-pVTZ value is $-14.5 \mathrm{kcal} / \mathrm{mol}$ which reduces to $-11.2 \mathrm{kcal} / \mathrm{mol}$ after the corresponding counterpoise correction, which is compared to -13.4 (or -11.4 when BSSE-corrected) and $-11.7 \mathrm{kcal} / \mathrm{mol}$ with the def2-TZVP or def2-QZVP, respectively. The $s_{6}$ parameterization is thus not expected to be significantly influenced by this basis set issue and would not significantly affect the association energies.

We also recognize at this stage that the corrections discussed so far are pairwise additive, which might be related to a slight tendency of BP86-D3 towards overbinding. A way to evaluate the (repulsive) amplitude of the three-body contribution is through the function [46]:

$$
E_{3-\text { body }}=C_{9}^{A B C} \frac{\left(3 \cos \theta_{a} \cos \theta_{b} \cos \theta_{c}+1\right)}{R_{A B} R_{B C} R_{A C}}
$$

where $A B C$ are all the atom triples, $\theta_{i}$ are the internal angles of the triangle 
formed by $R_{A B}-R_{B C}-R_{A C}$, and the coefficient $C_{9}^{A B C}$ is approximated by $C_{9}^{A B C}=-\sqrt{C_{6}^{A B} C_{6}^{B C} C_{6}^{A C}}$. While this contribution is believed to be negligible for small complexes, around $2 \%$ of the association energy of the benzene dimer [47], it might become crucial for larger systems, around $25 \%$ for two graphene layers [46]. In the present case, this contribution amounts for 1.2 $\mathrm{kcal} / \mathrm{mol}$ (which is 6-7 \% of the association energy if we take for instance the BP86-D3/CBS result of $-18.8 \mathrm{kcal} / \mathrm{mol}$ as reference for estimating the weight of this 3-body correction). This correction thus reduces the difference between the BP86-D2 and BP86-D3 results and appears not negligible. However, it is not expected to significantly influence the conclusions about the relative performance of methods and the derived association energies.

The relevance of the BP86-NL and B3P86-NL models was also assessed for the present non-covalent polyphenol dimer. In this case, the exchangecorrelation functional is defined by:

$$
E_{x c}[\rho]=E_{x}[\rho(\mathbf{r})]+E_{c}^{l o c a l}[\rho(\mathbf{r})]+E_{c}^{n o n-l o c a l}\left[\rho(\mathbf{r}), \rho\left(\mathbf{r}^{\prime}\right)\right]
$$

where $E_{x}$ is the B and B3 exchange parts, respectively, and $E_{c}^{\text {local }}\left(E_{c}^{\text {non-local }}\right)$ is P86 (VV10) in both functionals. Again the sequence of def2-xVP basis sets was used. The initial value imposed to the $b$ parameter for an efficient coupling of non-local correction with the exchange-correlation part was the available values for related models [48] i.e., $b=3.5(b=4.0)$ for BP86 (B3P86). Note that for the cases known up to now (BLYP vs. B3LYP and revPBE vs. revPBE0) the value of $b$ turns to be always lower for pure than for hybrid methods, as it should be upon a careful inspection of the whole function $\beta=\beta(b)$ entering into eq. (5). We then accordingly modified it in a systematic way to check the influence on the association energies. Table 2 presents the corresponding association energies, leading to the following 
conclusions: (i) going across the sequence def2-SVP/def2-TZVP/def2-QZVP largely reduces the BSSE in line with the observations made before; and (ii) the association energies depend on $b$ values, B3P86 always providing larger stabilization energies than BP86 at same $b$-value. Figure 4 exemplifies the amplitude of this variation for both BP86-NL and B3P86-NL models when the large def2-QZVP basis set is employed.

In the hope to obtain a refined value of $b$ for the use of this correction for polyphenol compounds, three systems (benzene-benzene, benzenemethanol, phenol-phenol, see Figure 5) were correspondingly selected. They are representative of the leading $\pi-\pi, \pi-\mathrm{OH}$, and $\mathrm{OH}-\mathrm{OH}$ interactions, respectively, as found in non-covalent polyphenol dimers. Note that very accurate $\mathrm{CCSD}(\mathrm{T}) / \mathrm{CBS}$ results are available in the literature for the association energy of these complexes of moderate size $[49,50]$, which will thus serve to guarantee the lowest possible deviation with respect to any benchmark thought. To do that, the Mean Absolute Deviation (MAD) is reduced as much as possible for association energies calculated at the BP86-NL and B3P86-NL levels, according to the $b$ values and with respect to the CCSD(T)/CBS association energies taken as reference. Note also that the large def2-QZVP basis set is used here to avoid any spurious BSSE. The following optimum $b=4.4$ and $b=5.1$ values were found, providing a MAD lower than 0.2 $\mathrm{kcal} / \mathrm{mol}$ in both cases with respect to the $\operatorname{CCSD}(\mathrm{T}) / \mathrm{CBS}$ results. We thus now predict with these optimized $b$ values, and again with the def2-QZVP basis set, association energies of -19.4 and $-17.0 \mathrm{kcal} / \mathrm{mol}$, with the BP86-NL and B3P86-NL models respectively, which are very close to the benchmark SCS-MP2-based results (Figure 3). 


\section{Conclusions}

The association or interaction energy of a large real-world non-covalent polyphenol dimer has been elucidated by dispersion-corrected DFT methods using several flavours. First, benchmark calculations at improved secondorder perturbation theory were performed to adequately bracket the stabilizing energy gained when the two monomers self-associate to form the complex. The use of large basis sets, up to the def2-QZVP, leads to sufficiently converged results. Interestingly, we estimate at the complete basis set limit (within an expected error bar or about $0.2 \mathrm{kcal} / \mathrm{mol}$ ) complexation energies of $-17.6 \mathrm{kcal} / \mathrm{mol}$ at both the BP86-D2 and BP86-B3 levels, the latter after taking into account the 3-body interactions, and of $-11.5 \mathrm{kcal} / \mathrm{mol}$ at the B3P86-D2 level. When these functionals combine in a purely ab initio fashion with a correlation correction (the VV10 functional) and with an optimum value for the attenuation $b$-parameter entering into this non-local functional, the values are -19.4 and $-17.0 \mathrm{kcal} / \mathrm{mol}$ (BP86-NL and B3P86-NL, respectively). All these schemes seem to slightly underestimate the SCS-S66-MP2based results although, however, it is satisfying to see that, even being fairly different in both the underlying density functional and the way in which they incorporate the missing dispersion forces, they predict enough stabilization energy to anticipate the existence of this kind of complexes almost independently of expected thermal or environmental conditions. Thus, a practical yet accurate combination of DFT-D2/DFT-D3 searches along potential energy hypersurfaces, thanks to rapid evaluation of gradients with these levels and their moderate scaling with size, together with refinements employing DFT-NL might constitute a valid strategy for further future studies. 


\section{Acknowledgements}

The work in Alicante is supported by the "Ministerio de Educación y Ciencia" of Spain and the "European Regional Development Fund" through project CTQ2011-27253. The work in Mons is supported by the Belgian National Fund for Scientific Research (FNRS). The work in Limoges is supported by the "Conseil Régional du Limousin" and COST actions FA1003 "East-West Collaboration for Grapevine Diversity Exploration and Mobilization of Adaptive Traits for Breeding" and CM0804 "Chemical Biology with Natural Products". The work in Malaysia is supported by Akademi Sains Malaysia through the SAGA grant C20 and by the Ministry of Higher Education through the grant 600-RMI/ST/FRGS 5/3/Fst (4/2011). The authors gratefully acknowledge the support by the Operational Program Research and Development for Innovation-European Regional Development Fund (project CZ.1.05/2.1.00/03.0058 of the Ministry of Education, Youth and Sports of the Czech Republic). I.B. gratefully thanks the "Association Djerbienne de France" (ADF) for the financial support.

\section{References}

[1] S. Quideau, Polyphénols Actualités (2006) 10.

[2] J. Buckingham (Ed.), Dictionary of Natural Products on DVD, Chapman \& Hall / CRC, London, 2011.

[3] V. Habauzit, C. Morand, Ther. Adv. Chronic. Dis. 3 (2012) 87.

[4] C. C. Tangney, H. E. Rasmussen, Curr. Atheroscler. Rep. 15 (2013) 324.

[5] F. Di Meo, J. C. Sancho-García, O. Dangles, P. Trouillas, J. Chem. Theory Comput. 8 (2012) 2034. 
[6] F. Nave, N. Bras, L. Cruz, N. Teixeira, N. Mateus, M. Ramos, F. Di Meo, P. Trouillas, O. Dangles, V. De Freitas, J. Phys. Chem. B 116 (2012) 14089.

[7] T. Tanaka, I Kouno, G. I. Nonaka, Biomimetic Synthesis and Related Reactions of Ellagitannins, in E. Poupon, B. Nay (Eds), Biomimetic Organic Synthesis, Wiley-VCH Verlag, Weinheim, 2011.

[8] J. Gorham, M. Tori, Y. Asakawa, The Biochemistry of the Stilbenoids, Chapman and Hall, London, 1995.

[9] S. S. Velu, N. F. Thomas, J.-F. F. Weber, Cur. Org. Chem. 16 (2012) 605 .

[10] S. S. Velu, F. Di Meo, P. Trouillas, J. C. Sancho-García, J.-F. F. Weber, J. Nat. Prod. 76 (2013) 538.

[11] S. S. Velu, I. Buniyamin, L. K. Ching, F. Feroz, I. Noorbatcha, L. C. Gee, K. Awang, I. A. Wahab, J.-F. F. Weber, Chem. Eur. J. 14 (2008) 11376 .

[12] C. Bonechi, S. Martini, A. Magnani, C. Rossi, Magn. Reson. Chem. 46 (2008) 625 .

[13] J. F. Dobson and T. Gould, J. Phys.: Condens. Matter 24 (2012) 073201.

[14] J. Klimeš and A. Michaelides, J. Chem. Phys. 137 (2012) 120901.

[15] S. Grimme, J. Comput. Chem. 27 (2006) 1787.

[16] Y. Liu and W. A. Goddard III, Mater. Trans. 50 (2009) 1664.

[17] J. Antony and S. Grimme, Phys. Chem. Chem. Phys. 8 (2006) 5287. 
[18] M. E. Foster and K. Sohlberg, Phys. Chem. Chem. Phys. 12 (2010) 307.

[19] S. Grimme, WIREs Comput. Mol. Sci. 1 (2011) 211.

[20] S. Grimme, J. Antony, S. Ehrlich, H. Krieg, J. Chem. Phys. 132 (2010) 154104 .

[21] S. Grimme, S. Ehrlich, L. Goerigk, J. Comput. Chem. 32 (2011) 1456.

[22] M. Dion, H. Rydberg, E. Schröder, D. C. Langreth, B. I. Lundqvist, Phys. Rev. Lett. 92 (2004) 246401.

[23] O. A. Vydrov and T. Van Voorhis, Phys. Rev. Lett. 103 (2009) 063004.

[24] K. Lee, É. D. Murray, L. Kong, B. I. Lundqvist, and D. C. Langreth, Phys. Rev. B 82 (2010) 081101.

H. Eshuis and F. Furche, J. Phys. Chem. Lett. 2 (2011) 983.

[25] O. A. Vydrov and T. Van Voorhis, J. Chem. Phys. 133 (2010) 244103.

[26] G. Román-Pérez and J. M. Soler, Phys. Rev. Lett. 103 (2009) 096102.

[27] P. Trouillas, C. Fagnere, R. Lazzaroni, C. Calliste, A. Marfak, J.-L. Duroux, Food Chem. 88 (2004) 571.

[28] P. Trouillas, P. Marsal, D. Siri, R. Lazzaroni, J.-L. Duroux, Food Chem. 97 (2006) 679.

[29] E. H. Anouar, P. Kosinova, D. Kozlowski, R. Mokrini, J.-L. Duroux, P. Trouillas, Phys. Chem. Chem. Phys. 11 (2009) 7659.

[30] E. H. Anouar, J. Gierschner, J.-L. Duroux, P. Trouillas, Food Chem. 131 (2012) 79 . 
[31] F. Neese, WIREs Comput. Mol. Sci. 2 (2012) 73.

[32] K. Eichkorn, O. Trutler, H. Öhm, M. Häser, R. Ahlrichs, Chem. Phys. Lett. 240 (1995) 283.

[33] F. Neese, F. Wennmohs, A. Hansen, U. Becker, Chem. Phys. 356 (2009) 98.

[34] J. Řezáč, K. E. Riley, P. Hobza, J. Chem. Theory Comput. 8 (2012) 4285 .

[35] K. E. Riley, M. Pitoňák, P. Jurečka, P. Hobza, Chem. Rev. 110 (2010) 5023.

[36] T. Schwabe, J. Comput. Chem. 33 (2012) 2067.

[37] S. Grimme, J. Chem. Phys. 118 (2003) 9095.

[38] T. Schwabe and S. Grimme, Acc. Chem. Res. 41 (2008) 569.

[39] C. R. Taylor, P. J. Bygrave, J. N. Hart, N. L. Allan, and F. R. Manby, Phys. Chem. Chem. Phys. 14 (2012) 7739.

[40] J. C. Sancho-García and Y. Olivier, J. Chem. Phys. 137 (2012) 194311.

[41] P. Hemberger, A. Bodi, C. Schon, M. Steinbauer, K. H. Fischer, C. Kaiser, and I. Fischer, Phys. Chem. Chem. Phys. 14 (2012) 11920.

[42] A. Karton, A. Tarnopolsky, J.-F. Lamère, G. C. Schatz abd Jan M. L. Martin, J. Phys. Chem. A 112 (2008) 12868.

[43] K. E. Riley, J. A. Platts, J. Řezáč, P. Hobza, J. Grant Hill, J. Phys. Chem. A 116 (2012) 4159. 
[44] S. Grimme, C. Mück-Lichtenfeld and J. Antony, J. Phys. Chem. C 111 (2007) 11199.

[45] T. Risthaus and S. Grimme, J. Chem. Theory Comput. 9 (2013) 1580.

[46] O. A. von Lilienfeld and A. Tkatchenko, J. Chem. Phys. 132 (2010) 234109 .

[47] S. Grimme, Chem. Eur. J. 18 (2012) 9955.

[48] W. Hujo and S. Grimme, Phys. Chem. Chem. Phys. 13 (2011) 13942.

[49] J. Řezáč, K. E. Riley, P. Hobza, J. Chem. Theory Comput. 7 (2011) 3466.

[50] M. S. Marshall, L. A. Burns, C. D. Sherrill, J. Chem. Phys. 135 (2011) 194102. 
- Table 1. List of parameters entering into the dispersion-corrected methods employed.

- Table 2. Association energies (in kcal/mol) at several non-local dispersioncorrected DFT levels, with the sequence of def2-xVP basis sets, as a function of the attenuation parameter. 
Table 1:

\begin{tabular}{lccccc}
\hline Method & $s_{6}$ & $s_{r, 6}$ & $s_{8}$ & $s_{r, 8}$ & $b$ \\
\hline BP86-D2 & 1.050 & 1.100 & - & - & - \\
B3P86-D2 & 0.780 & 1.100 & - & - & - \\
BP86-D3 & 1.000 & 1.139 & 1.683 & 1.000 & - \\
BP86-NL & - & - & - & - & 4.4 \\
B3P86-NL & - & - & - & - & 5.1 \\
\hline
\end{tabular}


Table 2:

\begin{tabular}{llccccc}
\hline & & \multicolumn{5}{c}{$\Delta E(\mathrm{kcal} / \mathrm{mol})$} \\
\cline { 3 - 7 } Method & Basis set & $b=3.5$ & $b=4.0$ & $b=4.5$ & $b=5.0$ & $b=5.5$ \\
\hline BP86-NL & def2-SVP & -38.5 & -32.3 & -27.4 & -23.5 & \\
& def2-TZVP & -32.0 & -25.5 & -20.5 & -16.4 & \\
& def2-QZVP & -29.9 & -23.5 & -18.5 & -14.4 & \\
B3P86-NL & def2-SVP & & -35.6 & -30.8 & -26.9 & -23.6 \\
& def2-TZVP & & -28.6 & -23.6 & -19.6 & -16.3 \\
& def2-QZVP & & -26.7 & -21.7 & -17.7 & -14.4 \\
\hline
\end{tabular}


- Figure 1. Chemical structure of the studied compound. The hydrogen atoms and corresponding $\mathrm{C}-\mathrm{H}$ bonds have been omitted for clarity. Figure created with ChemDraw.

- Figure 2. Optimized structure of the studied dimer from perpendicular (right) and side (left) views. Figure created with VMD.

- Figure 3. BSSE-uncorrected association energies (in kcal $/ \mathrm{mol}$ ) at several dispersion-corrected DFT levels, and with the sequence of def2$\mathrm{xVP}(\mathrm{x}=\mathrm{T}, \mathrm{Q})$ basis sets. Figure created with Xmgrace.

- Figure 4. BSSE-uncorrected association energies (in $\mathrm{kcal} / \mathrm{mol}$ ) at several non-local dispersion-corrected DFT levels, with the def2-QZVP basis set, as a function of the attenuation parameter. Figure created with Xmgrace.

- Figure 5. Selected interacting dimers for benchmarking. From top to bottom: Benzene-Benzene, Benzene-Methanol, and Phenol-Phenol. Figure created with VMD. 


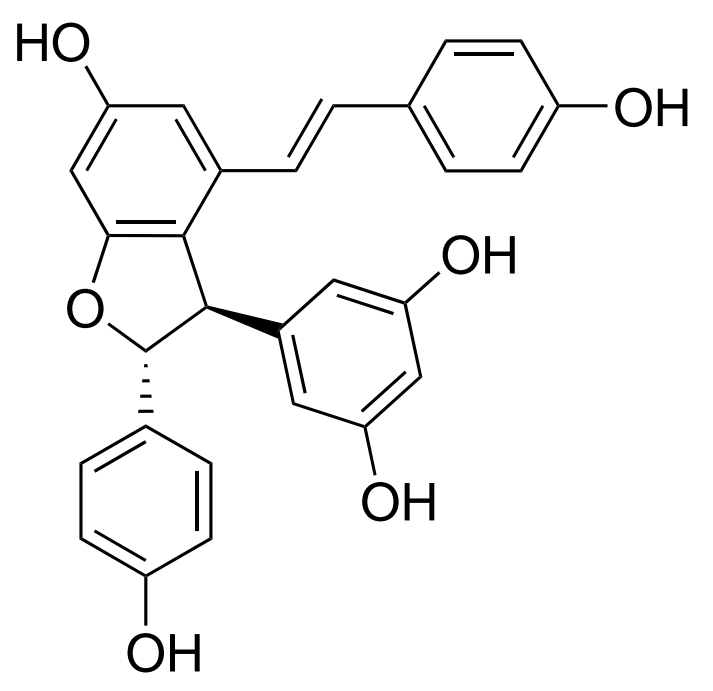

Figure 1. 

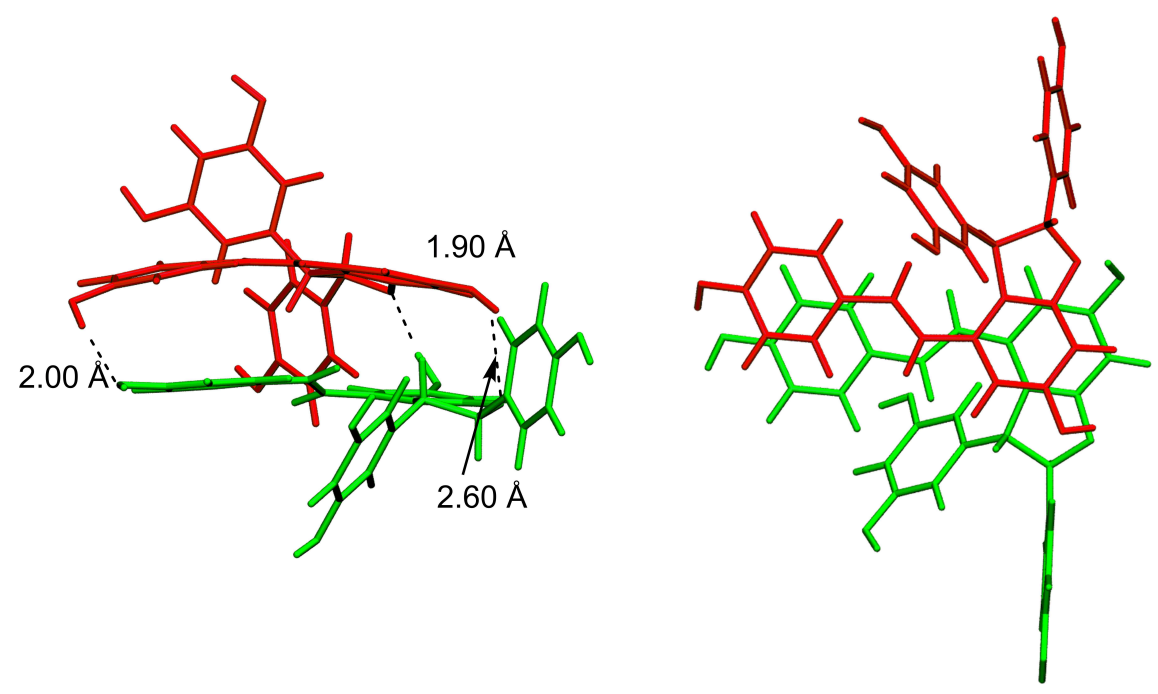

Figure 2. 


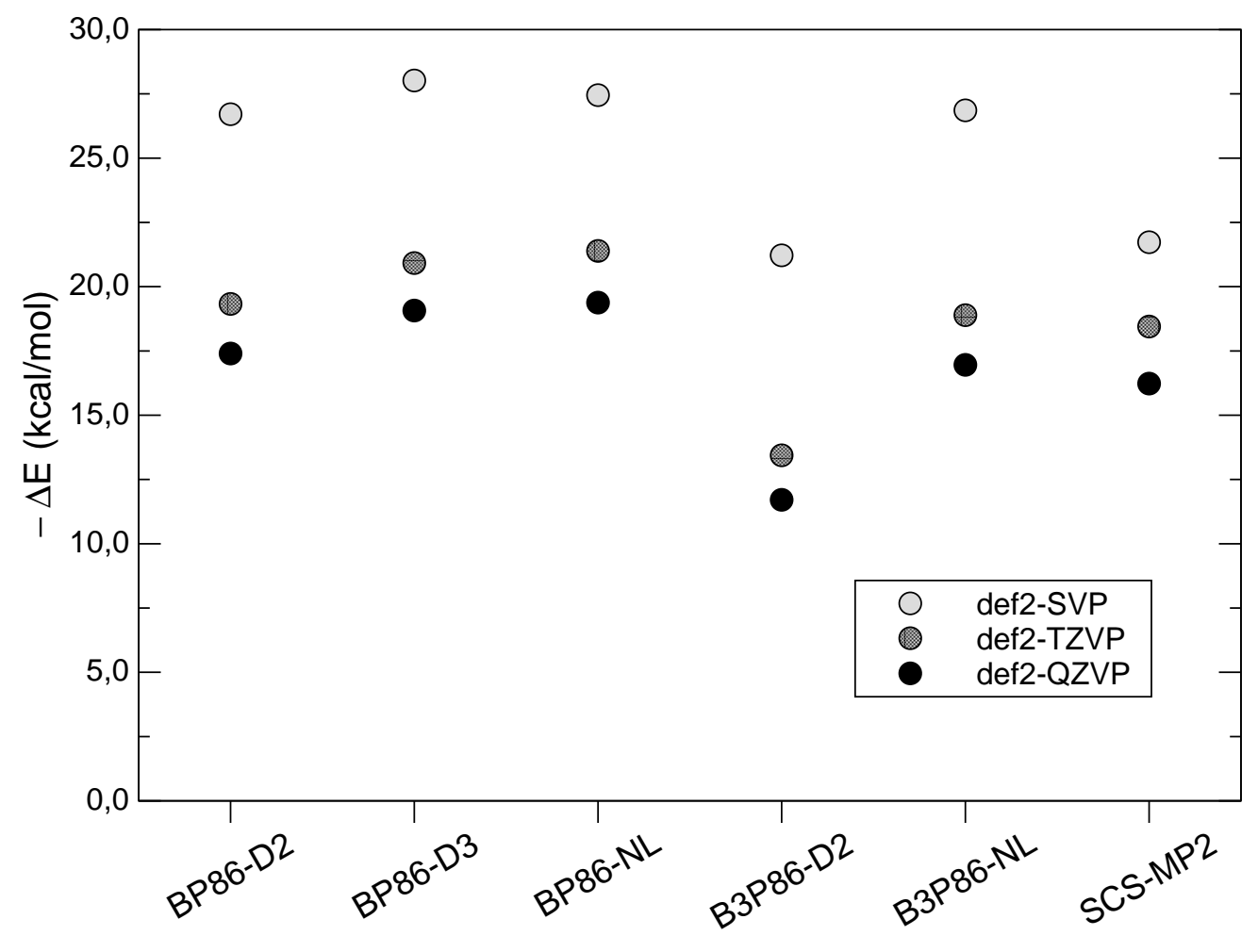

Figure 3. 


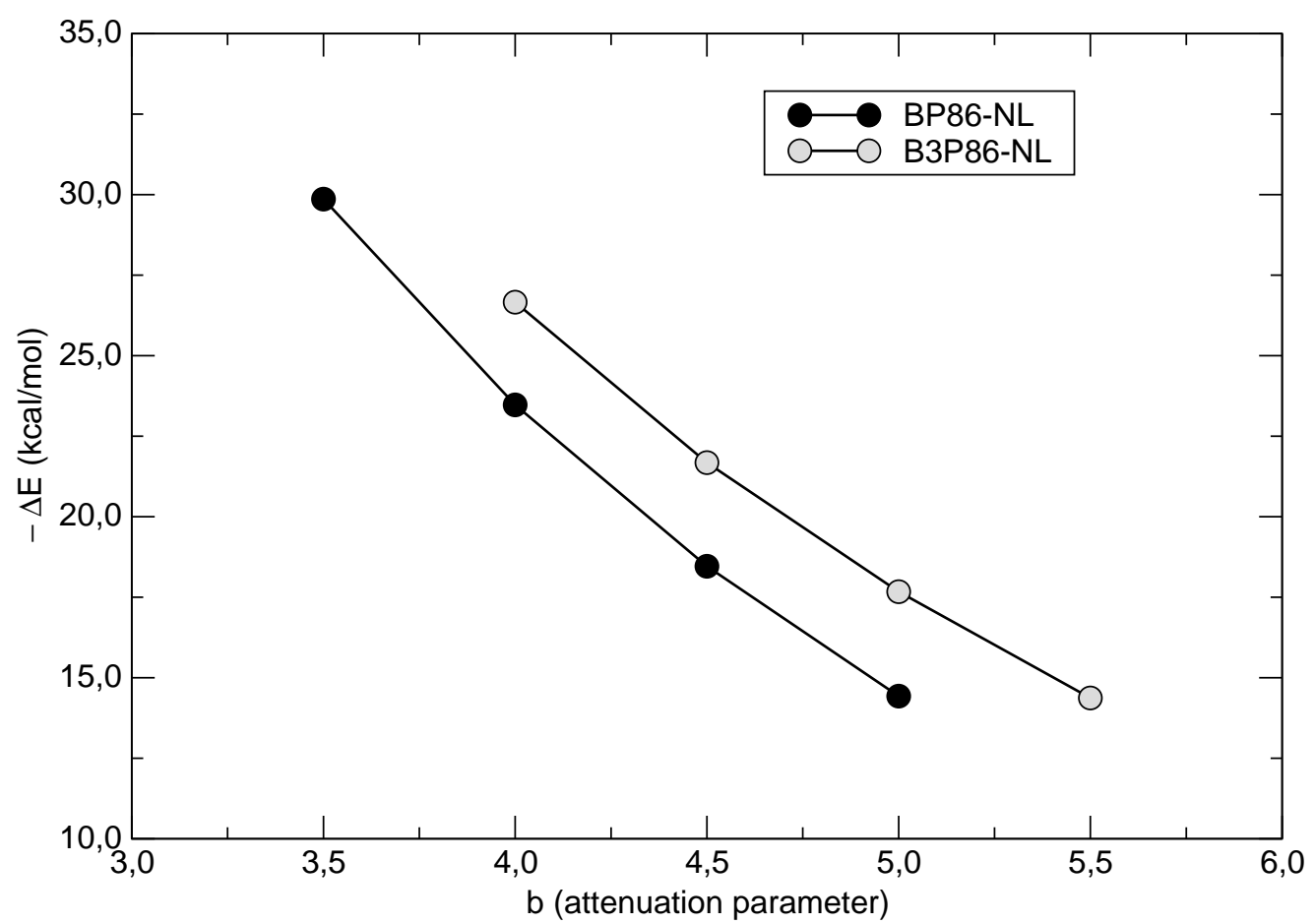

Figure 4 . 

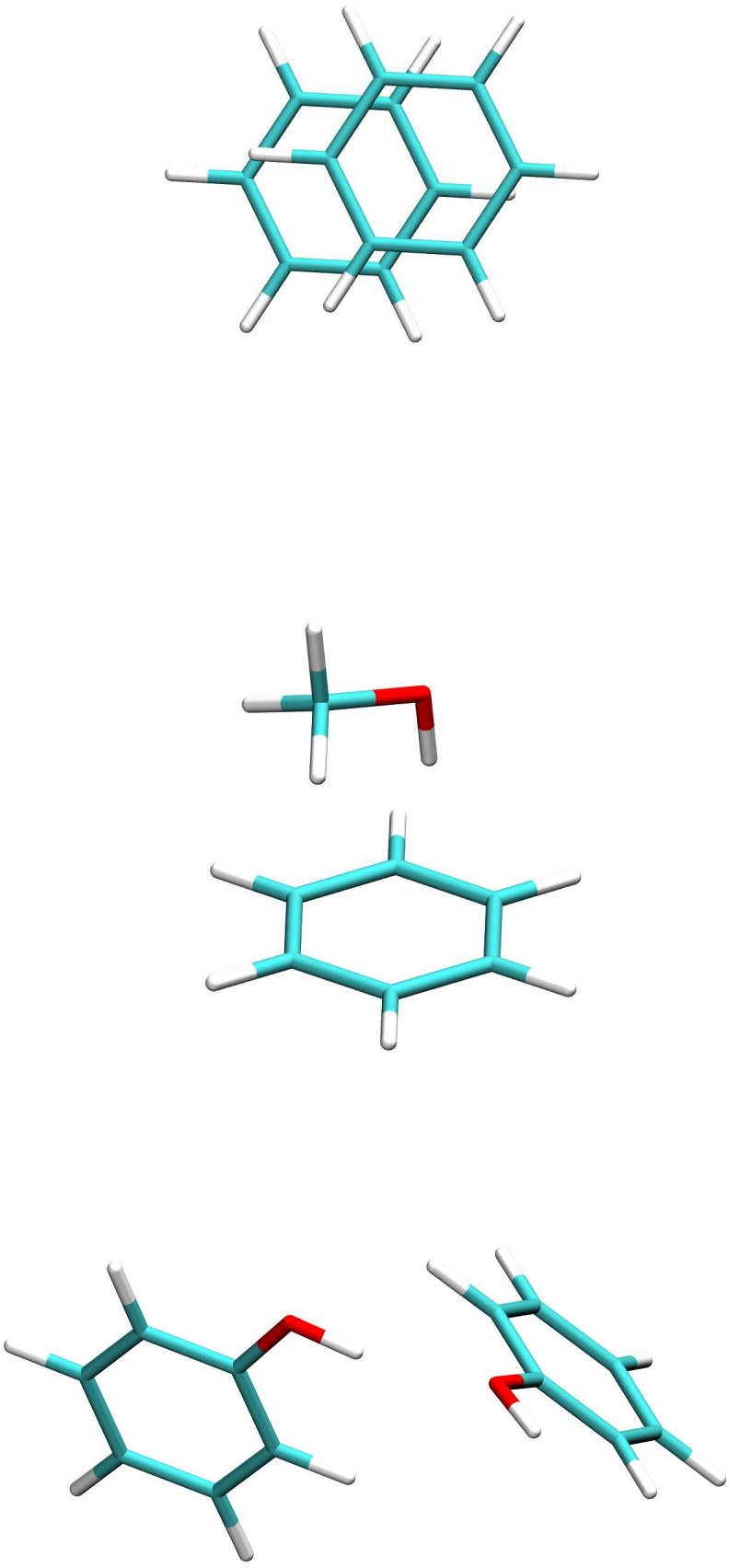

Figure 5 . 